\section{Autotransplantation of cryopreserved ovarian tissue in oncological patients: recovery of ovarian function}

\author{
Raffaella Fabbriं ${ }^{* 1}$, Gianandrea Pasquinelli², Valentina Magnani', Maria Macciocca', \\ Rossella Vicenti', Isabella Parazza', Roberto Paradisi', Cesare Battaglia', Stefania Rossi' \\ \& Stefano Venturoli'
}

\begin{abstract}
Aim: To present preliminary results of autotransplantation of cryopreserved ovarian tissue performed at Sant'Orsola-Malpighi Hospital, Bologna, Italy. Materials \& methods: Orthotopic transplantation was performed in two women with colorectal and breast cancer, and heterotopic transplantation was performed in one Hodgkin's lymphoma woman. The presence of micrometastasis in the ovarian tissue was checked, and morphological features of ovarian tissue were evaluated before transplantation. Ovarian function was monitored by hormonal and ultrasound-color Doppler examination after transplantation. Results: In all three women, no micrometastasis was found; light and transmission electron microscopy showed well-preserved thawed ovarian tissue. Ovarian function recovery was observed 2-4 months after transplantation. Spontaneous menstrual cycles occurred in two women with normal follicular densities. No periods occurred in the woman with low follicular density at the time of tissue collection. Conclusion: Ovarian tissue cryopreservation and transplantation is a promising approach for preserving ovarian function in women with cancer.
\end{abstract}

\section{Background}

Recent advances in the diagnosis of cancer, and the introduction of new protocols of chemo- and radio-therapy, have significantly increased the survival rates of children, adolescents and adults with cancer. However, these treatments are gonadotoxic and can severely affect or totally destroy the reproductive potential of patients. Women receiving chemotherapy and/or radiotherapy are likely to experience premature ovarian failure [1,2]. The increased life expectancy given to patients by the use of anticancer treatments can only be considered a success if the patient's quality-of-life is effectively respected.

Ovarian tissue cryopreservation represents a valid strategy to preserve reproductive function and steroidogenic activity in patients with a high risk of premature ovarian failure. This is a great alternative to oocyte cryopreservation because it can be performed at any time in the ovarian cycle, thereby avoiding delays in starting therapy; it is particularly indicated in patients with hormonesensitive tumors. In addition, ovarian tissue cryopreservation is the only available option to preserve ovarian function in prepubertal patients.

After thawing, the ovarian cortical strips may be grafted onto orthotopic sites, such as the atrophic ovary or pelvic peritoneum, close to infundibulopelvic ligaments or ovarian fossa, allowing the recovery of ovarian function and spontaneous pregnancy; or in heterotopic sites, such as the

'Gynaecology \& Pathophysiology of Human Reproduction Unit, Sant'Orsola-Malpighi Hospital, via Massarenti 13, 40138 Bologna, DIMEC, University of Bologna, Italy

${ }^{2}$ Clinical Pathology, Sant'Orsola-Malpighi Hospital, via Massarenti 9, 40138 Bologna, DIMES, University of Bologna, Italy

*Author for correspondence: Tel.: +39 0516363732; Fax: +39 0516363366; raffaella.fabbri@unibo.it

\section{KEYWORDS}

- fertility preservation - orthotopic/heterotopic transplantation • ovarian function recovery • ovarian tissue cryopreservation
Future
Medicine
part of 
subcutaneous space of the forearm or abdominal wall, allowing recovery of endocrine function and assuming the retrieval of mature oocytes for in vitro fertilization [3]. The efficiency of orthotopic and heterotopic transplantation of cryopreserved ovarian tissue has been established in terms of ovarian function recovery [4], and, to date, globally, 24 healthy babies have been born after orthotopic transplantation of cryopreserved ovarian tissue and four pregnancies are ongoing [5].

Herein we describe the first three cases of orthotopic and heterotopic transplantation of cryopreserved ovarian tissue carried out in the Gynaecology and Pathophysiology of Human Reproduction Unit, Sant'Orsola-Malpighi Hospital of Bologna, Italy. Patient 1 suffering from colorectal cancer, patient 2 suffering from breast cancer and patient 3 suffering from Hodgkin's lymphoma.

\section{Materials \& methods}

\section{- Freezing \& thawing procedures}

An ovarian biopsy from both ovaries was obtained by laparoscopy according to Paradisi et al. [6], and immediately transferred to the laboratory in fresh phosphate-buffered saline (PBS) medium supplemented with 10\% human serum (HS; provided by the Transfusion Centre of Sant'Orsola-Malpighi Hospital [Bologna, Italy]), in ice. The cortical tissue was dissected in strips $( \pm 1 \mathrm{~cm} \times 2 \mathrm{~mm} \times 1 \mathrm{~mm})$, and slowly frozen according to the protocol described by Fabbri et al. [7]. The cortical strips were rapidly thawed by a modified protocol described by Fabbri et al. [8]; that is, by removing cryoprotectants at $4^{\circ} \mathrm{C}$.

\section{- Light \& transmission electron microscopy} Light microscopy

One fragment $( \pm 1 \mathrm{~cm} \times 2 \mathrm{~mm} \times 1 \mathrm{~mm})$ from each ovary was fixed at the time of laparoscopy (fresh tissue) and, after thawing in $4 \%$ formaldehyde, embedded in paraffin and serially sectioned at a thickness of $4 \mu \mathrm{m}$.

Nine serial sections were obtained per block: the first, third and seventh were stained with hematoxylin and eosin (Merck, Darmstadt, Germany) to assess the presence of malignant cells in the ovarian tissue and the morphological features of follicles and stromal cells [9].

The second, fourth and sixth sections were incubated with different antibodies for immunohistochemistry to detect the presence of micrometastasis in the ovarian tissue, according to the
WHO criteria [10]. The following monoclonal mouse antihuman antibodies were used: CAM5.2 (1:20; Becton Dickinson, CA, USA) to detect cells originating from colorectal cancer; CAM5.2 and WT1 (1:50; Novocastra, Newcastle Upon Tyne, UK) to detect breast cancer cells; Ki-67 (1:230; Novocastra ${ }^{\mathrm{TM}}$, Newcastle upon Tyne, UK) and CD30 (1:40; Dako, Glostrup, Denmark) to detect Reed-Sternberg cells in Hodgkin's lymphoma. Dewaxed tissue sections were treated with citrate buffer, $\mathrm{pH}$ 6.0, at $120^{\circ} \mathrm{C}, 1$ atmospheric pressure for $21 \mathrm{~min}$ to unmask antigens; after blocking endogenous peroxidase with NovocastraPeroxidase Block, the sections were processed with a nonbiotin-amplified method (NovoLink ${ }^{\mathrm{TM}}$ Polymer Detection System, Novocastra), as previously described [11].

The fifth, eighth and ninth sections were collected (Superfrost-plus slides Menzel-Gläser, Braunschweig, Germany) for terminal deoxynucleotidyl transferase dUTP nick end labeling (TUNEL) analysis using the cell death detection kit conjungated with horseradish peroxidase (Roche, Mannheim, Germany) to estimate the percentage of apoptotic cells in the fresh and thawed ovarian tissue and, thus, to evaluate if apoptosis was involved in the follicle loss after freezing/thawing.

\section{Transmission electron microscopy}

Transmission electron microscopy analysis was performed on fresh and thawed ovarian samples as part of routine laboratory analysis involving patients who undergo ovarian tissue cryopreservation. One fragment from each ovary was fixed at the time of laparoscopy (fresh tissue) and after thawing in $4 \%$ paraformaldehyde, postfixed in 1\% buffered osmium tetroxide and embedded in epoxy resin (Araldite ${ }^{\circledR} \mathrm{M}$ hardener; Sigma-Aldrich, MO, USA), and then sectioned with an ultramicrotome (Ultracut; Reichert, Vienna, Austria). For each sample, one $0.5-\mu \mathrm{m}$ thick section out of every $30 \mu \mathrm{m}$ was collected and stained with toluidine blue to determine the follicular density (follicle number per $\mathrm{mm}^{2}$ of the overall section area). After semithin sectioning, the block was trimmed and 60-nm thick sections were collected on 200-mesh grids, stained with uranyl acetate lead citrate and observed with a Philips $410 \mathrm{~T}$ transmission electron microscope at $80 \mathrm{kV}$ to evaluate the ultrastructural features of follicles and stromal cells after cryopreservation [8]. 


\section{- Orthotopic transplantation procedure}

On the day of transplantation, cortical strips were thawed. Two or three strips were stitched together with 4-0 Vicryl ${ }^{\circledR}$ (Ethicon Inc., NJ, USA) by a biologist, by passing the needle between the stroma and cortex (Figure 1A) at $4^{\circ} \mathrm{C}$, and then rapidly transferred to the operating room in fresh PBS medium supplemented with $10 \%$ HS.

Ovarian tissue transplantation was performed by four-port laparoscopy: the primary $12-\mathrm{mm}$ port was inserted in the supraumbilical region for the $12-\mathrm{mm}$ telescope, $5-\mathrm{mm}$ ports were inserted in both the left and right lower quadrants at the same level lateral to the rectus sheath, and a fourth $12-\mathrm{mm}$ port was inserted in the midline in the suprapubic. A longitudinal incision of approximately $1 \mathrm{~cm}$ was made into the surface of both the right and left ovary, and a pocket was developed in the ovarian parenchyma by blunt dissection. Cortical strips were sutured into the ovarian
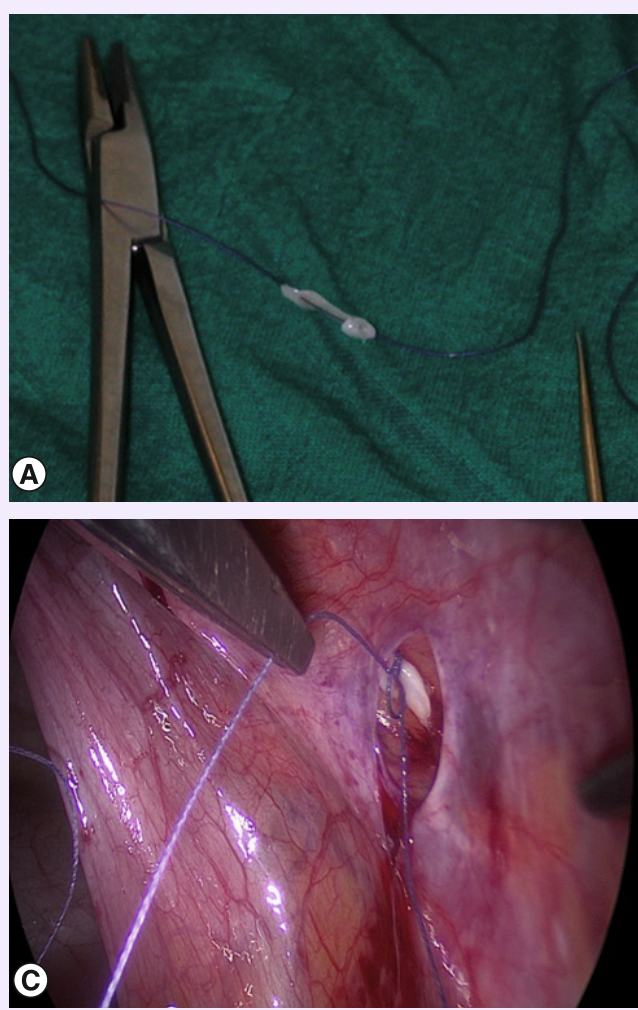

pocket and the same sutures were used to close the ovary, taking care not to cause ischemia of the tissue (Figure 1B).

To increase the chances of ovarian function recovery, a peritoneal pocket was created, through a $1.5-\mathrm{cm}$ incision close to the ovarian vessels and fimbria, on the right and/or left side. Then cortical strips were gently placed in the peritoneal pocket; the pocket was closed with a 4-0 Vicryl suture (Figure 1C).

\section{- Heterotopic transplantation procedure}

On the day of transplantation, cortical strips were thawed and immediately transferred to the operating room in fresh PBS medium supplemented with $10 \%$ HS.

A transverse incision of the skin of about $2 \mathrm{~cm}$ was performed in the suprapubic area on the right and left sides, and two subcutaneous pockets were created above the fascia. The cortical strips were gently placed individually in each pocket (Figure 1D). Finally, the pockets were closed with
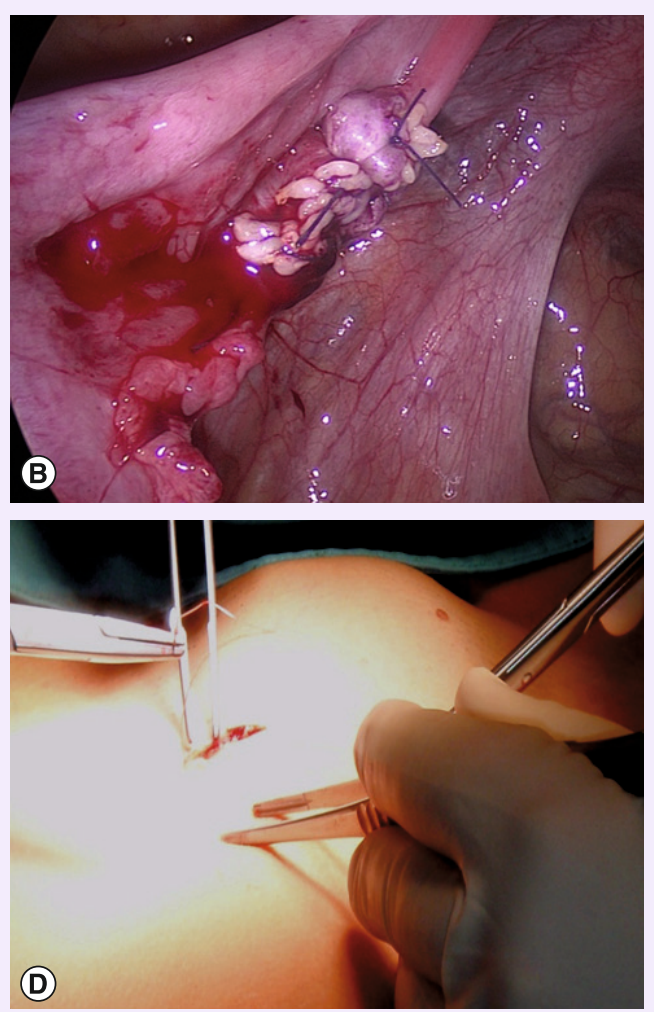

Figure 1. Surgical techniques of orthotopic and heterotopic transplantation of frozen-thawed ovarian strips. For orthotopic transplantation, the ovarian strips were (A) stitched together and transplanted at the (B) ovarian and (C) peritoneal sites. (D) For heterotopic transplantation, the ovarian cortical strips were gently inserted individually into the pockets created in the subcutaneous space of the abdominal wall. 
a 4-0 Vicryl suture and the skin sutured with a colorless intradermal 4-0 Vicryl suture.

\section{- Follow-up post-transplantation}

After transplantation, ovarian function was monitored by hormonal assay and ultrasound, and color Doppler examination at intervals of approximately 3 weeks.

\section{Hormonal assay}

All serum measurements were performed at the Central Laboratory of Sant'OrsolaMalpighi Hospital. Concentrations of folliclestimulating hormone (FSH), luteinizing hormone (LH), estradiol, progesterone and testosterone were assayed using an electrochemiluminescent method (Modular; Roche). Concentrations of antimullerian hormone $(\mathrm{AMH})$ were determined by ELISA using an AMH Gen II ELISA kit (Beckman Coulter Inc., CA, USA).

\section{D ultrasonographic \& color Doppler examination}

All subjects underwent an ultrasonographic examination of the uterus and ovaries using a multifrequency transvaginal transducer RIC5-9H (Voluson 730 Expert Sonography System; GE Healthcare Ultrasound, Zipf, Austria). The endometrial thickness and cyclical changes were reported and the ovarian volume and number and maximum diameter of the follicles recorded.

After ultrasonographic scanning, the patients were transvaginally evaluated with a multifrequency color Doppler system (Voluson 730 Expert Sonography System Color Doppler; GE Healthcare Ultrasound) for Doppler flow measurements of the main ovarian and intraovarian vessels. The pulsatility index (PI), defined as the difference between the peak systolic and enddiastolic flow divided by the mean maximum flow velocity, was electronically calculated by the machine. No significant differences between the PIs of the left and right side for the main ovarian and ovarian stromal arteries were observed; therefore, the average value of the arteries on both sides was used.

For heterotopic graft examination, a highresolution volumetric linear transducer (RSP16 multi-frequency $4 \mathrm{D}$ linear array transducer, Voluson 730 Expert Sonography System; GE Healthcare Ultrasound) was used and the ovarian tissue volume and number and maximum diameter of the follicles were recorded.
The Doppler flow of the main tissutal arteries was measured and PIs recorded.

\section{Results}

\section{- Quality control of frozen/thawed ovarian} tissue

A total of 92 follicles were assessed in fresh ( $\mathrm{n}=$ 51) and thawed $(n=41)$ samples: 25 fresh and 20 thawed in patient 1 , four fresh and three thawed in patient 2 , and 22 fresh and 18 thawed in patient 3 . The percentage of morphologically normal follicles was not significantly different between fresh $(81.6 \pm 10.2 \%)$ and thawed tissue $(73.8 \pm 19.8 \%)$. After thawing, approximately $30 \%$ of follicles showed oocytes with emptylooking cytoplasm, in which no mitochondria were distinguishable. This alteration was also present in $10 \%$ of fresh follicles.

After thawing, stromal cells presented a homogeneous distribution, and no vacuolization or edema was observed (Figure 2A \& B).

Follicles showing normal morphology after thawing presented ultrastructural features similar to those of fresh follicles (Figure 2C \& D): oocytes disclosed nuclei with a regular shape, dispersed chromatin and intact nuclear membrane, perinuclear cluster of mitochondria with normal matrices, and cytoplasm with homogeneous texture; granulosa cells also presented a normal chromatin pattern and cytoplasm density. After thawing, stromal cells showed dispersed chromatin or small chromatin clumps at the nuclear periphery and cytoplasm fragmentation.

TUNEL analysis did not show a significant difference between fresh and frozen-thawed samples. In cryopreserved ovarian tissue, $21 \pm$ $9.2 \%$ of stromal cells and $27 \pm 11.6 \%$ of granulosa cells showed TUNEL-positive nuclei, while in fresh tissue, the percentage was lower $(15 \pm$ $7.8 \%$ and $19 \pm 10.5 \%$, respectively) (Figure $2 \mathrm{E}$ \&F).

\section{- Patient history \& results after transplantation}

Patient 1

This patient was diagnosed with colorectal cancer Dukes B2 at the age of 23 years. In December 2003, she underwent rectal resection, and from February to August 2004, she received 12 cycles of chemotherapy (fluorouracil and folic acid) and posterior pelvic ( $45 \mathrm{~Gy}$ ) and boost $(54 \mathrm{~Gy}$ ) radiotherapy. In March 2004, before radiotherapy and after three cycles of chemotherapy, the patient signed written informed consent for ovarian tissue 


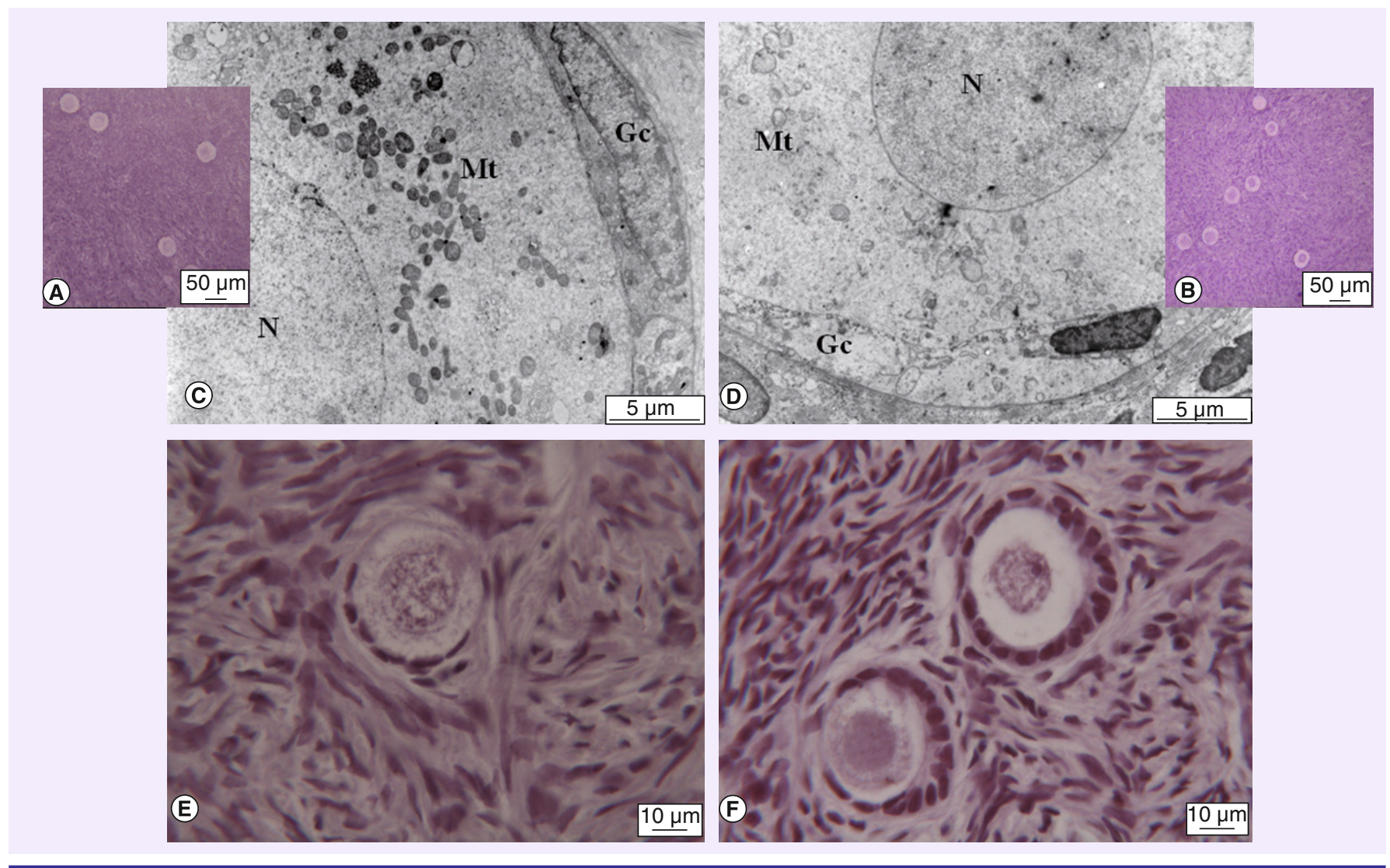

Figure 2. Representative images of the morphological and ultrastructural characteristics of fresh and frozen-thawed ovarian cortex of the three patients. Light microscopy: (A) fresh and (B) frozen-thawed ovarian cortex stained with toluidine blue. Transmission electron microscopy: (C) fresh and (D) frozen-thawed primordial follicle. (E) Fresh and (F) frozen-thawed ovarian cortex stained with terminal deoxynucleotidyl transferase dUTP nick end labeling.

Gc: Granulosa cell; MT: Mitochondria; N: Nucleus.

cryopreservation and this was performed within a few days. AMH level assessment $(5.4 \mathrm{ng} / \mathrm{ml})$ indicated an ovarian reserve according to chronological age. After chemo- and radio-therapy, the patient became amenorrheic and the concentrations of FSH (226 mIU/ml), LH (47 mIU/ml), estradiol $(<12 \mathrm{pg} / \mathrm{ml})$, testosterone $(<0.1 \mathrm{ng} / \mathrm{ml})$ and AMH (undetectable) confirmed premature ovarian failure. In 2008, the patient started hormone replacement therapy, which was stopped in 2010 owing to the presence of a fibroadenoma in the left breast. After its removal, the patient was stated to be disease free and she requested the replanting of frozen ovarian tissue.

She restarted hormonal therapy until a few days before transplantation in December 2011, 7 years after ovarian tissue cryopreservation. Vaginal sonography showed small and atrophic ovaries (right ovary: $0.4 \mathrm{~cm}^{3}$; left ovary: $0.3 \mathrm{~cm}^{3}$ ) where no antral follicles were visible. The mean ovarian artery PI was 4.16. The hormonal status confirmed castration: FSH: $134.6 \mathrm{mIU} / \mathrm{ml}, \mathrm{LH}$ : $62.9 \mathrm{mIU} / \mathrm{ml}$ and estradiol $<12 \mathrm{pg} / \mathrm{ml}$ (Figure 3). Testosterone levels were $<0.1 \mathrm{ng} / \mathrm{ml}$ and $\mathrm{AMH}$ levels were undetectable.

Histological and immunohistochemical analysis revealed the presence of eight follicles per $\mathrm{mm}^{2}$ in thawed ovarian tissue and the absence of malignant cells in fresh and frozen ovarian tissue.

On the day of transplantation, 20 cortical strips ( $40 \%$ of total amount of frozen tissue) were thawed: five strips were sutured into each ovarian pocket and five were gently placed in the right and left peritoneal pockets.

The first signs of restoration of ovarian function were observed 11 weeks after transplantation by an increase in estradiol $(78 \mathrm{pg} / \mathrm{ml})$ levels and a decrease in FSH $(90.4 \mathrm{mIU} / \mathrm{ml})$ and $\mathrm{LH}(34.5$ $\mathrm{mIU} / \mathrm{ml}$ ) levels, although they were still high (Figure 3). Vaginal sonography revealed follicular development in both ovarian sites: two follicles 

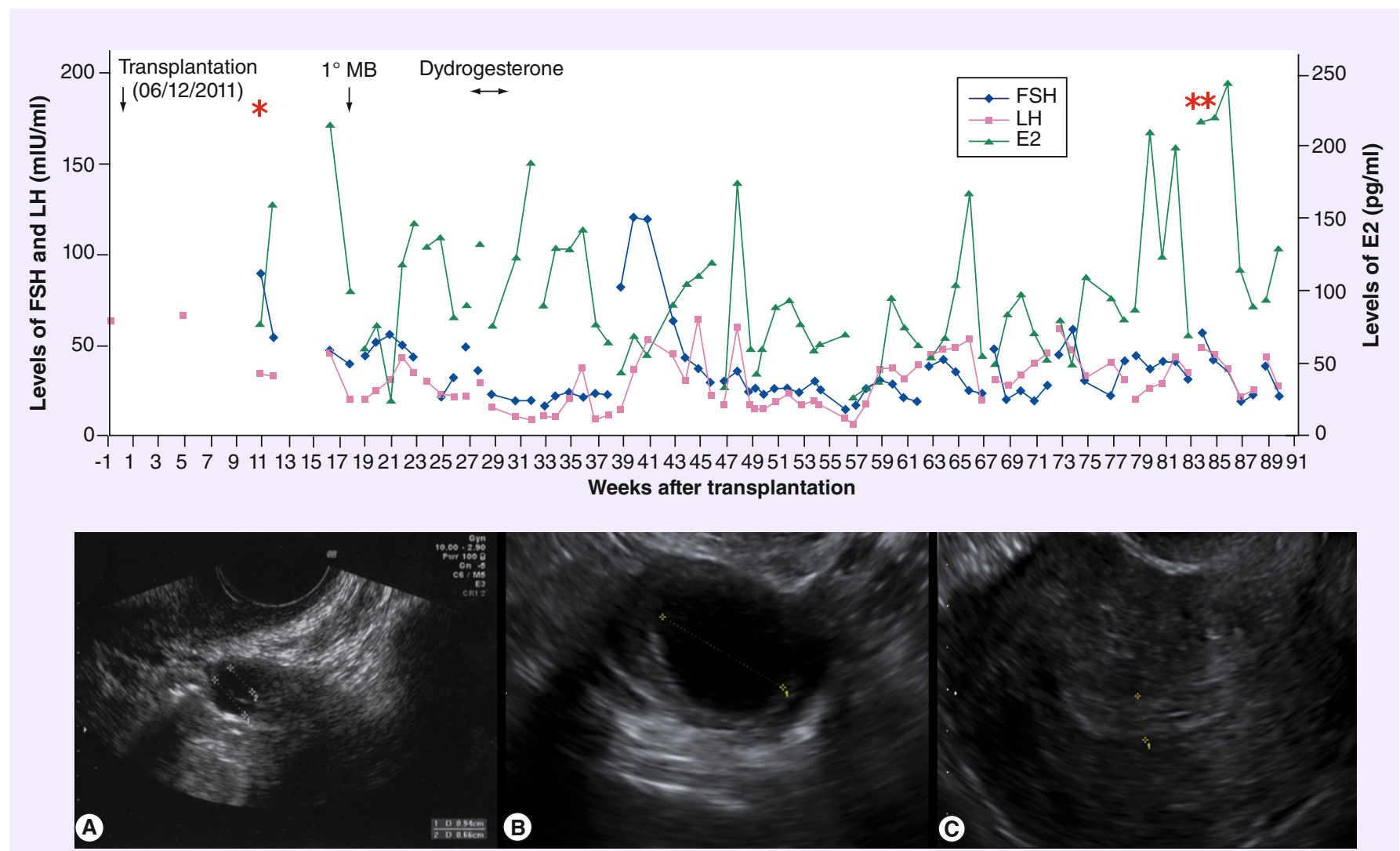

Figure 3. Patient 1: follicle-stimulating hormone, luteinizing hormone and estradiol concentrations after orthotopic transplantation. Asterisk: (A) transvaginal ultrasound 11 weeks after transplantation showing two follicles of 6-and 9-mm diameter in the left ovary. Double asterisk: transvaginal ultrasound performed at 11th day of the menstrual cycle showing (B) a follicle of 17-mm diameter and (C) trilaminar endometrium.

E2: Estradiol; FSH: Follicle-stimulating hormone; LH: Luteinizing hormone; MB: Menstrual bleeding.

( 6 and $9 \mathrm{~mm}$ ) in the left ovary (Figure $3 \mathrm{~A}$ ) and one $(5 \mathrm{~mm})$ in the right. At this time, the mean ovarian artery PI decreased to 2.14. The first spontaneous menstrual bleeding occurred exactly 18 weeks after transplantation. Subsequently, the patient's menstrual cycle was monitored with hormonal assays at intervals of around 7 days. The levels of FSH and LH continued to decrease (Figure 3), but the levels of progesterone remained low in the luteal phase (0.25-0.6 $\mathrm{ng} / \mathrm{ml})$. Testosterone levels increased to $0.4 \mathrm{ng} /$ $\mathrm{ml}$, returning within the reference range, while AMH levels remained undetectable.

In the following 8 weeks, the patient had no further menstruation, therefore dydrogesterone (10 mg/day) was administered for 1 week, after which she had her second menstrual cycle. FSH and LH concentrations declined further (19.4 $\mathrm{mIU} / \mathrm{ml}$ and $10.7 \mathrm{mIU} / \mathrm{ml}$, respectively) and the estradiol concentration was $124 \mathrm{pg} / \mathrm{ml}$. The left ovarian artery PI still decreased to 1.87 , whereas the right ovarian artery presented a PI value of 2.49. The patient's third menstruation occurred without medication 26 days after the previous one. After that, the patient continued to have menstrual cycles every 30-40 days with normal menstrual flow (Figure 3B \& C), and bilateral tubal patency was demonstrated by sonosalpingography. 91 weeks after ovarian tissue transplantation, follicular development was still ongoing; however, no pregnancy had been conceived.

During the follow-up post-transplantation, progesterone levels were always low in the luteal phase and never exceeded $1.89 \mathrm{ng} / \mathrm{ml}$; testosterone levels remained stable at around $0.3-0.4 \mathrm{ng} /$ $\mathrm{ml}$, and AMH levels were undetectable.

Trends in this patient's FSH, LH and estradiol concentrations are shown in Figure 3.

\section{Patient 2}

In September 2005, this patient was diagnosed with infiltrating lobular carcinoma of the left 
breast at the age of 34 years. She underwent quadrantectomy and axillary node dissection (pT1c pN1 mi [sn] pMx G1, positive for estrogen [90\%] and progesterone [30\%] receptors).

The patient had to be treated with four cycles of doxorubicin and cyclophosphamide chemotherapy, boost (60 Gy) radiotherapy, trastuzumab for 1 year and leuprorelin and tamoxifen for 2 years. Since the patient did not want to preclude the possibility of a future pregnancy (AMH levels of $1.2 \mathrm{ng} / \mathrm{ml}$ suggested a reduced follicular reserve), she requested the cryopreservation of her ovarian tissue. After signed written informed consent, ovarian tissue cryopreservation was performed before anticancer treatments.

In July 2008, the patient completed her treatment. After this, she had irregular menstrual cycles that definitively ceased in March 2011. In June 2012, 6.5 years after the ovarian tissue cryopreservation, the decision to transplant her ovarian tissue was taken. Ultrasound evaluation showed small ovaries (right ovary: $0.6 \mathrm{~cm}^{3}$; left ovary: $0.4 \mathrm{~cm}^{3}$ ) without antral follicles. The mean ovarian artery PI was 4.56. Hormonal assessment (FSH: $86.1 \mathrm{mIU} / \mathrm{ml}$; LH: $46.3 \mathrm{mIu} /$ $\mathrm{ml}$; estradiol $<12 \mathrm{pg} / \mathrm{ml}$ (Figure 4); testosterone $<0.1 \mathrm{ng} / \mathrm{ml}$; undetectable AMH) confirmed her menopausal status.

Histological and immunohistochemical analysis of fresh and thawed ovarian tissue with CAM 5.2 and WT1 did not identify malignant cells.

Because the follicular density was very low (one follicle per $\mathrm{mm}^{2}$ ), it was decided to transplant all cryopreserved cortical slices. Therefore, on the day of the transplantation, 46 cortical strips were thawed: 30 strips were sutured into the left ovarian pocket, 13 were sutured into the right ovarian pocket and the remaining three strips were gently placed in a peritoneal pocket on the right side.

10 weeks after transplantation, FSH and LH levels decreased to 37.8 and $30.0 \mathrm{mIU} / \mathrm{ml}$, respectively; estradiol remained low $(<12 \mathrm{pg} /$ $\mathrm{ml}$ ) (Figure 4). Testosterone levels increased to $0.2 \mathrm{ng} / \mathrm{ml}$ and AMH levels remained undetectable. Transvaginal sonography showed the presence of two follicles ( 6 and $7 \mathrm{~mm}$ ) on the right ovary (Figure 4A), two (6 and $15 \mathrm{~mm}$ ) on the left ovary and one of $15 \mathrm{~mm}$ in the right peritoneal window (Figure 4B). The mean ovarian artery PI was 2.11. Follow-up examination showed hormonal values almost unchanged and follicular development in both orthotopic sites, although follicles grew slowly and never reached a diameter greater than $4-6 \mathrm{~mm} .61$ weeks after transplantation (August 2013), ovarian activity ceased. The patient has never menstruated.

Trends in this patient's FSH, LH and estradiol concentrations are shown in Figure 4.

\section{Patient 3}

In December 2002, this woman who was then 29 years old was diagnosed with Hodgkin's lymphoma stage III, mixed-cellularity subtype (CD30+, CD15+/-). After signed written informed consent, ovarian tissue cryopreservation was undertaken before she received cancer treatments. Subsequently, the patient received six cycles of doxorubicin, bleomycin, vinblastine and dacarbazine chemotherapy, and supradiaphragmatic (92 Gy) and abdominal radiotherapy (67 Gy). She also underwent laparotomy to remove abdominal lymph nodes. Before anticancer treatments, AMH levels were $3.6 \mathrm{ng} / \mathrm{ml}$. After chemo-/radio-therapy, she had irregular menstrual cycles alternating with amenorrhea; however, she had two spontaneous pregnancies, giving birth to two healthy babies, 2 and 4 years after the end of treatment.

Since 2009, the patient's periods have definitively ceased and she presented pronounced menopausal symptoms (hot flushing and severe osteoporosis). In March 2012, she requested autotransplantation of cryopreserved ovarian tissue. Her menopausal status was confirmed by vaginal ultrasound showing atrophic ovaries (right ovary: $1.7 \mathrm{~cm}^{3}$; left ovary: $2.2 \mathrm{~cm}^{3}$; antral follicles not detectable) and by hormonal assessment showing elevated levels of FSH (113.5 $\mathrm{mIU} / \mathrm{ml})$ and $\mathrm{LH}(40.1 \mathrm{mIU} / \mathrm{ml})$, low levels of estradiol $(<12 \mathrm{pg} / \mathrm{ml})$ (Figure 5) and testosterone $(<0.1 \mathrm{ng} / \mathrm{ml})$, and undetectable levels of AMH. The mean ovarian artery PI was 3.99. Because the patient was interested only in endocrine function recovery, and the presence of adhesions due to the previous surgery could hinder laparoscopy, heterotopic transplantation of ovarian tissue was proposed.

The analysis of the ovarian tissue showed the presence of eight follicles per $\mathrm{mm}^{2}$ and the absence of malignant cells. In particular, immunohistochemical staining with anti-CD30 and -ki67 antibodies did not disclose Reed-Sternberg cells in the ovarian tissue.

On the day of the transplantation, ten cortical strips (36\% of total amount of frozen tissue) 


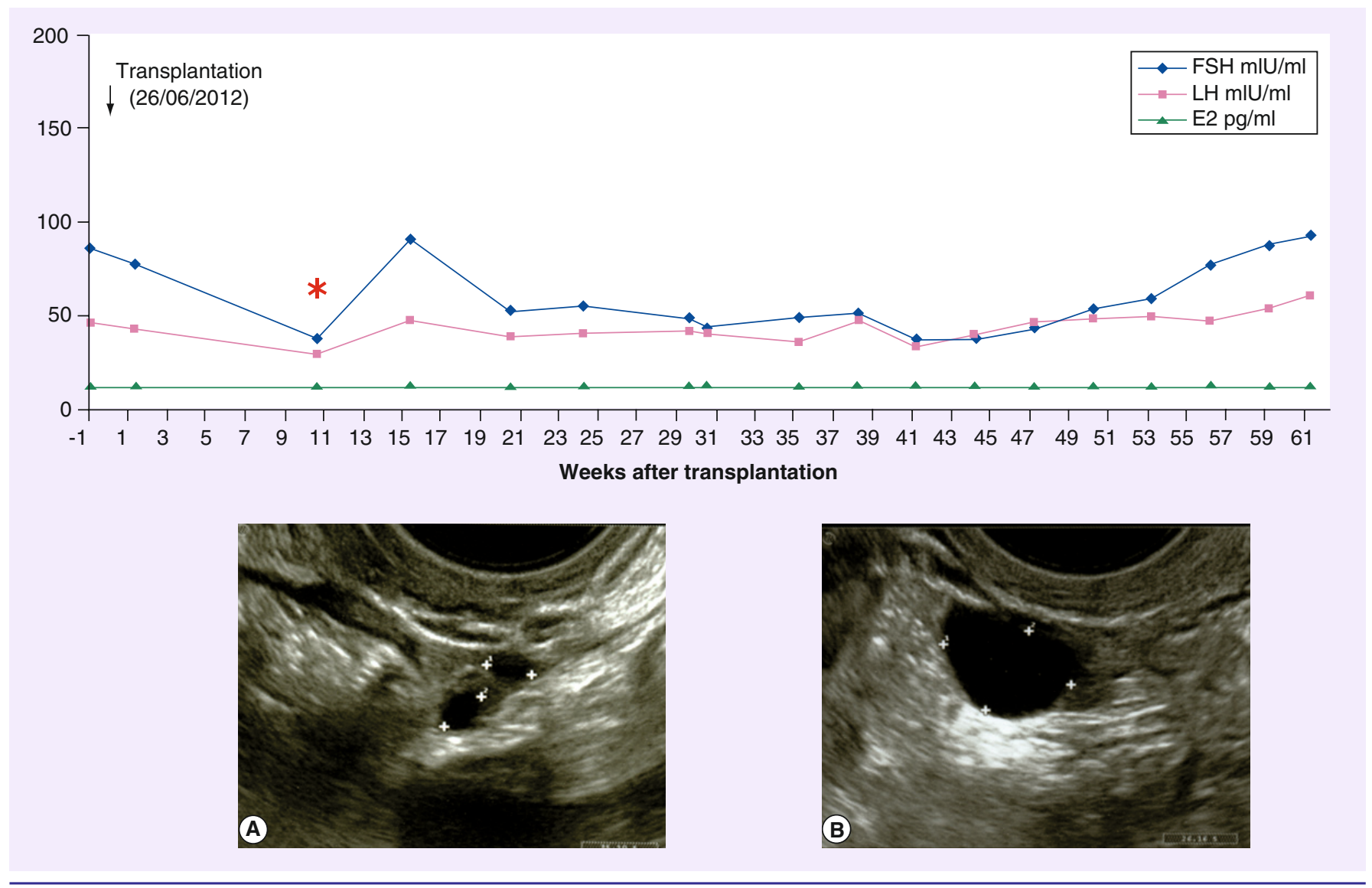

Figure 4. Patient 2: follicle-stimulating hormone, luteinizing hormone and estradiol concentrations after orthotopic transplantation. Asterisk: (A) transvaginal ultrasound 10 weeks after transplantation showing two follicles of 6 - and 7-mm diameter in the right ovary and (B) one follicle of 15-mm diameter in the right peritoneal window.

E2: Estradiol; FSH: Follicle-stimulating hormone; LH: Luteinizing hormone.

were thawed and five strips each were placed in the right and left subcutaneous pockets.

At approximately 4 weeks after transplantation, sonographic examination revealed no follicular development, but did reveal a discrete vascularization in the left graft (PI: 1.96). 19 weeks after ovarian tissue grafting, menopausal symptoms ceased and the patient reported breast tenderness and the presence of bulges in the area of transplantation. Follicular development was observed in both subcutaneous sites of the graft: four follicles $(4,6,6$ and $8 \mathrm{~mm})$ in the right graft (Figure 5A) and five follicles $(2,3$, 4,4 and $4 \mathrm{~mm}$ ) in the left graft. The Doppler flow supported further vascular improvement: right graft mean artery PI: 1.42; left graft mean artery PI: 1.24. Subsequently, FSH and LH levels dropped to 9.4 and $11.1 \mathrm{mIU} / \mathrm{ml}$, respectively, and estradiol levels increased to 526.9 $\mathrm{pg} / \mathrm{ml}$. Ultrasound examinations and hormonal levels were encouraging and it was decided to wait for the spontaneous resumption of the menstruation, also in light of previous literature data [12], which reported the resumption of menstruation 8.5 months after heterotopic replanting. 33 weeks after the transplant, she had her first spontaneous menstruation and a transvaginal ultrasound (performed 2 weeks later) revealed the development of two follicles $(3$ and $14 \mathrm{~mm})$ in the left atrophic ovary (Figure 5B). Subsequently, and until August 2013, the patient had periods approximately every 26 days, with normal menstrual flow. Hormonal levels (FSH, LH, estradiol, progesterone and testosterone) remained within the premenopausal reference range, while AMH levels were always undetectable. 76 weeks after ovarian tissue transplantation, follicular development was still ongoing in the graft sites. Trends in this patient's FSH, LH and estradiol concentrations are shown in Figure 5.

A bone mineral density test was requested for the patient in order to evaluate if the recovery of steroidogenic function could have also improved 
the osteoporosis condition, but the result of the exam was not ready at the end of the study (August 2013).

\section{Discussion}

This report describes our experience and first results obtained after orthotopic and heterotopic transplantation of cryopreserved ovarian tissue.

Since 2002, the ovarian tissue of 380 pediatric and adult patients has been cryopreserved at our unit (Gynaecology and Pathophysiology of Human Reproduction Unit, Sant'OrsolaMalpighi Hospital). Ovarian tissue cryopreservation requests have increased considerably in recent years; however, despite such a large number of patients, to date, only a few have requested ovarian tissue reimplantation. This is because most of these patients are still receiving treatment or still have to complete the followup period of at least 5 years between anticancer treatments and reimplantation. In addition, some patients did not experience premature ovarian failure and others have not yet required the ovarian tissue reimplantation.

Before transplantation, we evaluated tissue quality and viability of thawed ovarian samples, since a well-preserved ovarian tissue is a prerequisite for further follicle maturation. According to other recent studies [13,14], light and transmission electron microscopy showed a good preservation of most thawed follicles and stromal cells. Degenerated empty-looking oocytes were observed in thawed tissue ( 30\%): this finding was believed to be a consequence of organelle swelling and rupture, as a result of osmotic stress induced by the cryopreservation procedure [14,15]. Oocytes with similar morphology were also seen in fresh tissue (10\%), raising the possibility that this feature could also be related to dysfunctional follicologenesis. No signs of increased apoptosis
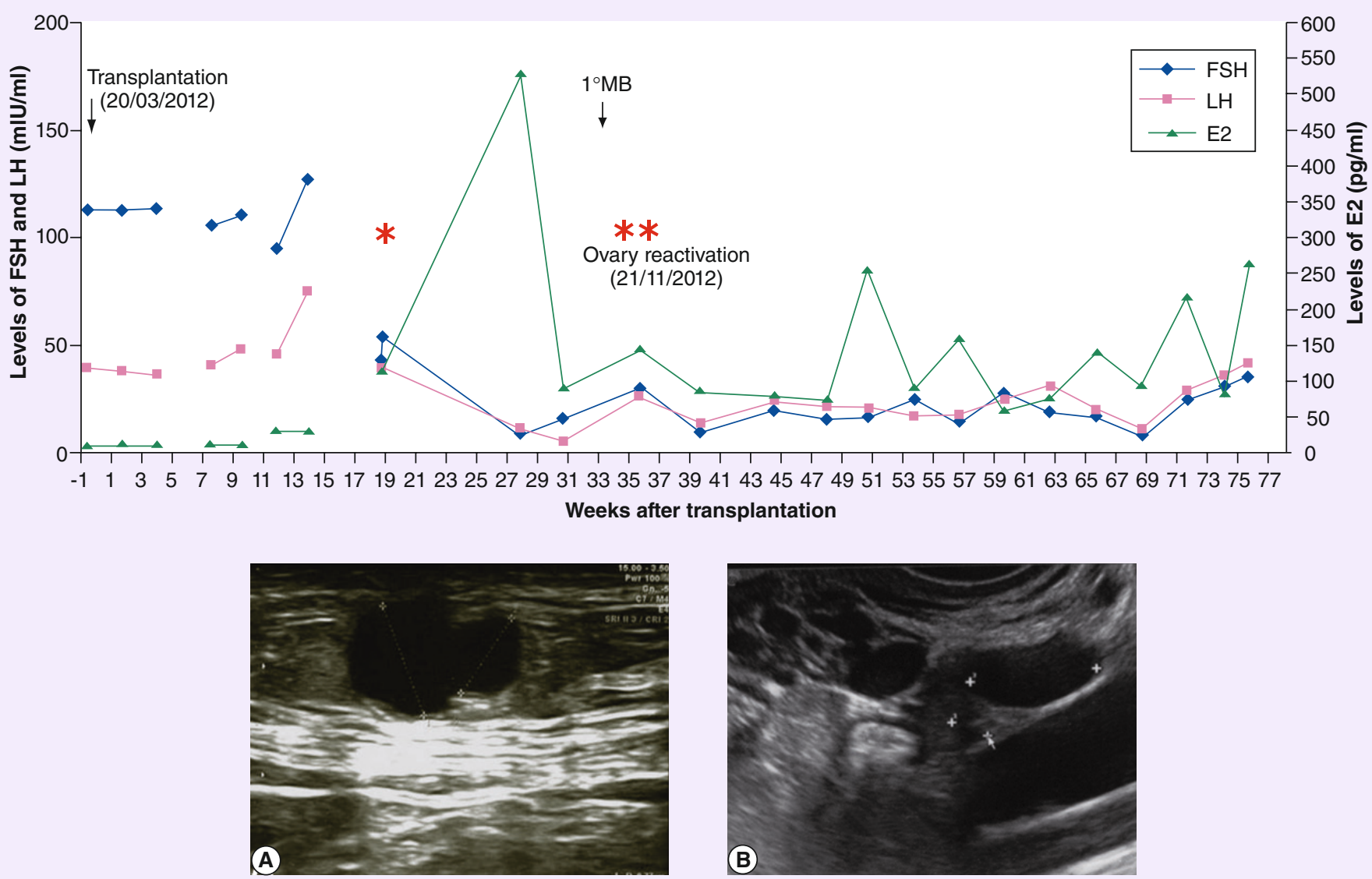

Figure 5. Patient 3: follicle-stimulating hormone, luteinizing hormone and estradiol concentrations after heterotopic transplantation. Asterisk: (A) transabdominal ultrasound 19 weeks after transplantation showing two follicles of 6- and 8-mm diameter in the right heterotopic site. Double asterisk: (B) transvaginal ultrasound 35 weeks after transplantation showing two follicles of 3-and 14-mm diameter in the left ovary. E2: Estradiol; FSH: Follicle-stimulating hormone; LH: Luteinizing hormone; MB: Menstrual bleeding. 
were observed after thawing, suggesting that apoptosis does not play a predominant role in cell degeneration after cryopreservation [14].

No evidence of malignant cell contamination was observed in patients' ovarian tissue. According to the most recent review, the risk of reintroducing malignant cells was estimated to be moderate in patients 1 and 2, and low in patient 3 [16]. However, even for those cancers in which the risk is low, the presence of metastasis in the ovary cannot be ruled out, and a careful analysis must always be carried out to assess the safety of the transplantation procedure.

As literature data showed the recovery of ovarian function and the birth of healthy babies from reimplanting cryopreserved ovarian tissue by one-step laparoscopy [17] or laparotomy [18-20], we performed the orthotopic transplantation by one-step laparoscopy to also avoid two anesthesias in a week and to limit the stress for patients.

Patient 1 resumed menstrual periods 18 weeks after transplantation; these recurred about every 30-40 days. However, high basal FSH levels during the follicular phase and low basal progesterone levels during the luteal phase persisted. Similar hormonal patterns after ovarian tissue transplantation have been found by other authors [21-23]. This finding might reflect a low number of surviving follicles in the graft, which could lead to a poor responder status after transplantation. The high levels of FSH might also be explained by the absence of inhibitory pathway, such as inhibin or AMH secretion, by growing follicles after transplantation. It is likely that chemotherapy before ovarian biopsy could contribute to a reduced follicular reserve in the patient.

Patient 2 resumed ovarian function 10 weeks after transplantation with decreases in FSH and LH levels, and follicular development in the graft sites. Although these first results were encouraging, subsequently, FSH values probably did not fall enough to restart the normal production of estradiol. The hormone values were compatible with a lack of recovery of normal ovarian activity. In fact, during follow-up monitoring, follicles grew slowly and did not reach ovulation. Ovarian activity ceased 61 weeks after transplantation and menstrual cycles never occurred. This result probably reflected a reduced follicular density at the time of tissue retrieval and underlines the importance of estimating the ovarian reserve before ovarian tissue cryopreservation, in particular for patients in their mid-30s, when the follicular reserve becomes compromised [5]. However, even in patients with reduced ovarian reserve, ovarian tissue cryopreservation might be considered, since coping with cancer with the hope of being able to fulfil the desire for motherhood plays a fundamental psychological role in the difficult process of care.

Heterotopic transplantation was performed in patient 3 who wished for the resumption of steroidogenic function. This procedure has the advantages of being noninvasive, being feasible when the presence of adhesions can preclude entry into the abdominal/pelvic area, and can be easily repeated when endocrine function stops [5]. A very recent study reported the first clinical pregnancy from heterotopic grafting of cryopreserved ovarian tissue and in vitro oocyte fertilization with intracytoplasmic sperm injection in a women after a bilateral oophorectomy for a granulosa cell tumor [3]. This result is very promising and offers optimism for cancer patients lacking a suitable ovarian site for transplantation.

After heterotopic transplantation, our patient experienced her first menstrual bleeding later than patient 1 (33 weeks after transplantation). It is possible that ovarian tissue replanting in the heterotopic site needs more time owing to differences in temperature, paracrine factors and blood supplies compared with the ovarian site. However, her menstrual periods occurred approximately every 26 days and the hormonal pattern returned to premenopausal levels, with an improvement of menopausal symptoms. A few weeks later, the patient also recovered activity in the menopausal ovary. This finding has previously been reported in one patient [24], and several hypotheses have been advanced [25]:

- Heterotopically reimplanted ovarian tissue might stimulate the regeneration of oocytes from the resident germ cells;

- Stem cells in the cryopreserved ovarian tissue might migrate from the heterotopic site to the ovary;

- Germ cells in the bone marrow, stimulated by the transplanted ovarian tissue, might migrate to the atrophic ovary via the bloodstream.

In summary, this study reports follicular development after orthotopic and heterotopic transplantation, according to previous studies $[5,26,27]$ and the expected time course for the 
development of primordial/primary follicles to the antral stage [28]. The phenomena were associated with a progressive recovery of normal vascularization in ovarian or subcutaneous transplanted tissues, and the resumption of steroidogenic activity, allowing an improvement of patients' menopausal symptoms and life quality. While there was a decrease in FSH and an increase in estradiol levels, AMH levels in our patients remained undetectable during the entire follow-up post-transplantation. This result was consistent with those of other studies [23,29], reporting undetectable or very low AMH levels after transplantation, even in patients at the time of conception. The low concentrations of AMH might likely reflect a perimenopausal status due to a low number of follicles in the transplanted tissue.

\section{Conclusion}

The analysis of the first cryopreserved ovarian tissue reimplantations showed that both procedures allow the restoration of ovarian function, as demonstrated by follicle development and hormonal serum levels. The ovarian medulla, the peritoneal pocket close to the ovarian hilus and the subcutaneous space of the abdominal wall resulted in efficient sites for reimplantation. Important predictive factors of the success of reimplantation are the patient's age at the time of laparoscopy and chemotherapy received before ovarian biopsy.

\section{Future perspective}

In the last decade, significant advances have been made in ovarian tissue cryopreservation, and the results obtained, in terms of ovarian activity resumption and pregnancies after transplantation of frozen-thawed ovarian tissue, are encouraging, and suggest that this procedure should be considered for fertility preservation and the elimination of the prospect of menopause for cancer patients.

Further research is needed to obtain the best results in terms of steroidogenic and reproductive function recovery. The optimization of the whole procedure could increase the tissue quality after thawing and reduce follicle loss after thawing and transplantation. In addition, the assessment of the

\section{EXECUTIVE SUMMARY}

\section{Aim of the study}

- The study describes preliminary results obtained from reimplantation of cryopreserved ovarian tissue carried out at the Gynaecology and Pathophysiology of Human Reproduction Unit, Sant'Orsola-Malpighi Hospital of Bologna, Italy.

\section{Materials \& methods}

- Orthotopic transplantation (on the atrophic ovary and peritoneal pocket), with regard to reproductive function restoration, was laparoscopically performed in two women with colorectal and breast cancer, and heterotopic transplantation (in the subcutaneous space of the abdominal wall), with regard to hormonal restoration, was performed in one woman with Hodgkin's lymphoma.

- Before grafting, morphological (histological and ultrastructural) features of thawed ovarian tissue and the presence of micrometastasis were carefully checked. After transplantation, ovarian function was monitored by hormonal assay and ultrasound-color Doppler.

\section{Results}

- Thawed ovarian tissue resulted well-preserved, and no micrometastasis was found.

- The resumption of ovarian function was observed 2 months after orthotopic transplantation and 4 months after heterotopic transplantation, as showed by decreasing follicle-stimulating hormone levels, increasing estradiol levels and follicle development in the grafts.

- Two patients resumed spontaneous menstrual cycles. No periods were observed in the patient with breast cancer, whose follicular density was very low at the time of tissue recovery.

\section{Conclusion}

- Ovarian tissue cryopreservation followed by transplantation (orthotopic and heterotopic sites) was found to be safe and applicable to restoring ovarian function in patients with premature ovarian failure. The ovarian reserve of the patient at the time of tissue retrieval and anticancer treatments (chemotherapy and/or radiotherapy) before cryopreservation may be important predictive factors for the success of the reimplantation. 
presence of metastasis should be always performed to avoid the risk of reintroducing malignant cells and make safe the autotransplantation of cryopreserved ovarian tissue. Histological and immunohistochemical techniques cannot guarantee the absence of micrometastasis, since malignant cells could be easily missed. For this reason, the analysis performed, its sensitivity and the results obtained should be extensively discussed with each patient. To date, to overcome current limits, molecular assessment should be performed.

\section{Acknowledgements}

The authors thank scrub nurse Liliana Ida Peccina who assisted the surgeon in the ovarian tissue transplantation with efficiency and competence.
Financial \& competing interests disclosure The authors have no relevant affiliations or financial involvement with any organization or entity with a financial interest in or financial conflict with the subject matter or materials discussed in the manuscript. This includes employment, consultancies, honoraria, stock ownership or options, expert testimony, grants or patents received or pending, or royalties.

No writing assistance was utilized in the production of this manuscript.

\section{Open Access}

This work is licensed under the Creative Commons Attribution-NonCommercial 3.0 Unported License. To view a copy of this license, visit http://creativecommons.org/ licenses/by-nc-nd/3.0/

\section{References}

Papers of special note have been highlighted as:

- of interest

-• of considerable interest

1 Larsen EC, Müller J, Schmiegelow K, Rechnitzer C, Andersen AN. Reduced ovarian function in long-term survivors of radiation- and chemotherapy-treated childhood cancer. J. Clin. Endocrinol. Metab. 88(11), 5307-5314 (2003).

2 Donnez J, Martinez-Madrid B, Jadoul P, Van Langendonckt A, Demylle D, Dolmans MM. Ovarian tissue cryopreservation and transplantation: a review. Hum. Reprod. Update 12(5), 519-535 (2006).

3 Stern CJ, Gook D, Hale LG, First reported clinical pregnancy following heterotopic grafting of cryopreserved ovarian tissue in a woman after a bilateral oophorectomy. Hum. Reprod. 28(11), 2996-2999 (2013).

4 Grynberg M, Poulain M, Sebag-Peyrelevade S, le Parco S, Fanchin R, Frydman N. Ovarian tissue and follicle transplantation as an option for fertility preservation. Fertil. Steril. 97(6), 1260-1268 (2012).

- Summarizes the state of the field of ovarian cryopreservation and transplantation, as well as the limits and future prospects of these new techniques.

5 Donnez J, Dolmans MM, Pellicer A et al. Restoration of ovarian activity and pregnancy after transplantation of cryopreserved ovarian tissue: a review of 60 cases of reimplantation. Fertil. Steril. 99(6), 1503-1513 (2013).

- Reports and discusses the results of 60 orthotopic reimplantations of cryopreserved ovarian tissue performed in three centers (Belgium, Denmark and Spain).
6 Paradisi R, Fabbri R, Magnani V, Battaglia C, Venturoli S. A new simple technique of laparoscopic temporary ovarian suspension in addition to ovarian cryopreservation for women prior to posterior pelvic radiation. Gynecol. Oncol. 117(2), 385-386 (2010).

7 Fabbri R, Venturoli S, D'Errico A et al. Ovarian tissue banking and fertility preservation in cancer patients: histological and immunohistochemical evaluation. Gynecol. Oncol. 89(2), 259-266 (2003).

8 Fabbri R, Pasquinelli G, Keane D, Magnani V, Paradisi R, Venturoli S. Optimization of protocols for human ovarian tissue cryopreservation with sucrose, 1,2-propanediol and human serum. Reprod. Biomed. Online 21(6), 819-828 (2010).

- Presents the optimization of the human ovarian tissue cryopreservation protocol, testing different concentrations of human serum and cryoprotectants (propanediol and sucrose) in the freezing and thawing solutions.

9 Fabbri R, Vicenti R, Magnani V et al. Cryopreservation of ovarian tissue in breast cancer patients: 10 years of experience. Future Oncol. 8(12), 1613-1619 (2012).

10 WHO. WHO Handbook for Reporting Results of Cancer Treatment (Offset Publication No. 48). WHO Press, Geneva, Switzerland, 16-21 (1979).

11 Simioniuc A, Campan M, Lionetti V et al. Placental stem cells pre-treated with a hyaluronan mixed ester of butyric and retinoic acid to cure infarcted pig hearts: a multimodal study. Cardiovasc. Res. 90(3), 546-556 (2011).

12 Stern CJ, Toledo MG, Hale LG, Gook DA, Edgar DH. The first Australian experience of heterotopic grafting of cryopreserved ovarian tissue: evidence of establishment of normal ovarian function. Aust. NZ J. Obstet. Gynaecol. 51(3), 268-275 (2011).

13 Nottola SA, Camboni A, Van Langendonckt A et al. Cryopreservation and xenotransplantation of human ovarian tissue: an ultrastructural study. Fertil. Steril. 90(1), 23-32 (2008).

14 Wang LH, Mullen SF, Li Y, Zhong JQ, Crister JK, Chen ZJ. Morphological and apoptotic comparison of primordial and primary follicles in cryopreserved human ovarian tissue. Reprod. Domest. Anim. 44(6), 879-883 (2009).

15 Camboni A, Martinez-Madrid B, Dolmans MM et al. Preservation of fertility in young cancer patients: contribution of transmission electron microscopy. Reprod. Biomed. Online 17(1), 136-150 (2008).

16 Dolmans MM, Luyckx V, Donnez J, Andersen CY, Greve T. Risk of transferring malignant cells with transplanted frozenthawed ovarian tissue. Fertil. Steril. 99(6), 1514-1522 (2013).

-. Systematic review of the studies evaluating the presence of malignant cells in cryopreserved human ovarian tissue. Based on the available data, the risk of ovarian tissue contamination has been estimated for the different cancer diseases.

17 Dittrich R, Lotz L, Keck G et al. Live birth after ovarian tissue autotransplantation following overnight transportation before cryopreservation. Fertil. Steril. 97(2), 387-390 (2012).

18 Silber SJ. Ovary cryopreservation and transplantation for fertility preservation. Mol. Hum. Reprod. 18(2), 59-67 (2012).

19 Meirow D, Levron J, Eldar-Geva T et al. Pregnancy after transplantation of cryopreserved ovarian tissue in a patient with 
ovarian failure after chemotherapy. N. Engl. J. Med. 353(3), 318-321 (2005).

Sánchez-Serrano M, Crespo J, Mirabet V et al. Twins born after transplantation of ovarian cortical tissue and oocyte vitrification. Fertil. Steril. 93(1), 268. e11-268.e13 (2010).

21 Donnez J, Squifflet J, Van Eyck AS et al. Restoration of ovarian function in orthotopically transplanted cryopreserved ovarian tissue: a pilot experience. Reprod. Biomed. Online 16(5), 694-704 (2008). Tryde Schmidt KL, Andersen CY, Starup J, Loft A, Byskov AG, Nyboe Andersen A. Orthotopic autotransplantation of cryopreserved ovarian tissue to a woman cured of cancer - follicular growth, steroid production and oocyte retrieval. Reprod. Biomed. Online 8(4), 448-453 (2004).
23 Greve T, Schmidt KT, Kristensen SG, Ernst E, Andersen CY. Evaluation of the ovarian reserve in women transplanted with frozen and thawed ovarian cortical tissue. Fertil. Steril. 97(6), 1394-1398 (2012).

24 Oktay K. Spontaneous conceptions and live birth after heterotopic ovarian transplantation: is there a germline stem cell connection? Hum. Reprod. 21(6), 1345-1348 (2006).

25 Oktay K, Türkçüo lu I, Rodriguez-Wallberg KA. Four spontaneous pregnancies and three live births following subcutaneous transplantation of frozen banked ovarian tissue: what is the explanation? Fertil. Steril. 95(2), 804.e7-804.e10 (2011).

26 Demeestere I, Simon P, Buxant F et al. Ovarian function and spontaneous pregnancy after combined heterotopic and orthotopic cryopreserved ovarian tissue transplantation in a patient previously treated with bone marrow transplantation: case report. Hum. Reprod. 21(8), 2010-2014 (2006).

27 Kim SS. Assessment of long term endocrine function after transplantation of frozenthawed human ovarian tissue to the heterotopic site: 10 year longitudinal follow-up study. J. Assist. Reprod. Genet. 29(6), 489-493 (2012).

28 Gougeon A. Regulation of ovarian follicular development in primates: facts and hypotheses. Endocr. Rev. 17(2), 121-155 (1996).

29 Janse F, Donnez J, Anckaert E, de Jong FH, Fauser BC, Dolmans MM. Limited value of ovarian function markers following orthotopic transplantation of ovarian tissue after gonadotoxic treatment. J. Clin. Endocrinol. Metab. 96(4), 1136-1144 (2011). 